\title{
RADIO STUDIES OF COSMIC RAYS IN NEARBY GALAXIES
}

\author{
E. HUMMEL \\ Nuffield Radio Astronomy Laboratories, University of Manchester, \\ Jodrell Bank, Macclesfield, Cheshire, SK11 9DL, England
}

\begin{abstract}
The constraints on cosmic ray electron sources and propagation as derived from radio continuum observations of galaxies are reviewed. Special attention is paid to the inferences which can be obtained from the radio continuum properties of spiral galaxies seen edge-on.
\end{abstract}

\section{INTRODUCTION}

The bulk of the radio continuum emission from spiral galaxies observed below a frequency of $10 \mathrm{GHz}$ is non-thermal of origin and as shown by the radio spectra and linearly polarized emission due to the synchrotron emission mechanism. This means that the radio emissivity is proportional to $\mathrm{n}_{e} \mathrm{~B}^{1-\alpha}$, where $\mathrm{n}_{e}$ is the density of relativistic electrons, B the magnetic field strength and $\alpha$ is the radio spectral index. The latter is directly related to $\gamma$, the energy index of the cosmic ray electrons ${ }^{1}$ : $\alpha=(\gamma-1) / 2$

This link between the radio continuum emission and cosmic rays, in particular the electron component, features in many studies of the large scale radio continuum structure of spiral galaxies. In short, these studies often focus on: $i)$ the sources of relativistic electrons, ii) the propagation of these electrons and iii) the B-field strength and configuration. Recent reviews of such studies have been given in Hummel (1990), Klein (1990), Krause (1990) and Beck (this volume) and I refer to these papers and the references given there for more details. Here I want to concentrate on some specific observational results which are important for studies on the propagation of the relativistic electrons.

${ }^{1} \alpha$ defined by $\mathrm{S} \propto \nu^{\alpha}$ and $\gamma$ is defined by $\mathrm{n}_{e} \propto \mathrm{E}^{\gamma}$ 
In Section 2 I discuss briefly the sources of the relativistic electrons. Section 3 deals with the radial distribution of the non-thermal and thermal emission in spiral galaxies. The emphasis is on the results obtained for the nearest face-on galaxy for which this can be studied, IC342. The largest fraction of this paper, Section 4, discusses the results obtained for the radio continuum emission from edge-on spiral galaxies, in particular NGC891 and NGC4631. Such galaxies are of course most suitable to study the propagation of the relativistic electrons perpendicular to the plane of a galaxy and the disk-halo interaction. Section 5 summarizes the more general results which constrain cosmic ray propagation models for galaxies.

\section{SOURCES OF RELATIVISTIC ELECTRONS}

Radio continuum observations can be used to discuss the sources of cosmic ray electrons. At the moment it appears as if the pendulum is near one of its extremes again: i.e. recent star formation is closely related to the origin of the relativistic electrons. The main reasons for me to adopt this view are $i$ ) the relation between the dust temperature and the radio emissivity in Sbc galaxies (Hummel et al. , 1988) and $i$ ) the results obtained from a comparison of the far infrared and radio continuum emission from isolated and interacting galaxies (Wunderlich, 1989). The often quoted tight correlation between the non-thermal radio continuum and the thermal dust emission in itself can in this respect only be used as a consistency argument.

Type II supernova remnants (SNRs) are often seen as the sources of the cosmic ray electrons and there is no compelling evidence based on radio continuum results against this view. On the other hand it is certainly not a necessary conclusion. The apparent objections: $i$ ) the deficiency by a factor 10 if one applies the $\Sigma$-D relation to explain the radio continuum emission, ii) the spectral index of the integrated nonthermal emission and iii) the "smooth" distribution of the non-thermal emission, can be countered by arguments that take into account lifetime and propagation effects. Assuming that SNRs are the sole source of cosmic ray electrons Hummel and van der Hulst (1986) found an efficiency of $1 \rightarrow 5 \%$ for SNRs in NGC4038/38 to produce cosmic rays (including relativistic electrons).

If the sources of the relativistic electrons are closely related to the more recent star formation then it is conceivable that these sources are distributed like the thermal gas. In principle this distribution can be determined from $\mathrm{H} \alpha$ measurements and multi-frequency radio observations. Another tracer of recent star formation is the thermal dust emission. Bicay et al. (1989) used the distribution of the $60 \mu \mathrm{m}$ emission to define the distribution of the cosmic ray sources. If on the other hand the sources are distributed like the older disk population the source distribution 
may be derived from optical photometry. As long as it is not known beyond doubt what the sources of cosmic rays are it is probably wise to consider both possibilities.

\section{THE RADIAL DISTRIBUTIONS}

The main tools provided by radio continuum measurements for the study of the cosmic rays are the brightness distributions at various frequencies (nowadays available at frequencies from $327 \mathrm{MHz} \rightarrow 10.7 \mathrm{GHz}$ ) and the resulting spectral index distributions. Usually the emission is averaged in rings in the plane of the galaxy, hence neglecting the fact that the radio emission in general is a function of radius and azimuthal angle, to obtain the radial brightness and spectral index distributions. It is of course essential to use measurements which have recorded all the emission. Because we do not have an a priori knowledge of the radio continuum structure this is not always so trivial as it seems.

The radio emission we observe is a mixture of thermal and non-thermal emission. This is fortunate but also a nuisance. Fortunate because it allows us to determine the distribution of the relativistic electron sources (provided they are related to recent star formation). A nuisance because in order to study the propagation of cosmic ray electrons the radial brightness and spectral index distributions need to be corrected for the thermal contribution. Note in this respect that the differences in the diffusion models Segalovitz (1977) and van der Kruit (1977) obtained for M51 arose because of the difference in their evaluation of the thermal emission.

The thermal emission distribution and the corrected non-thermal emission distributions are usually obtained from measurements at (only) two frequencies with the assumption of a constant non-thermal spectral index $\left(\alpha_{n t h}\right)$ out to a certain radius. The choice of $\alpha_{n t h}$ is somewhat arbitrary, however it can be constrained by comparing the resulting thermal emission with $\mathrm{H} \alpha$ measurements and with high frequency radio measurements. If $\alpha_{n t h}$ is slowly decreasing (spectrum steepening) with radius then the assumption of a constant $\alpha_{n t h}$ will lead to an underestimate of the exponential scale length of the thermal emission. However, no attempts have been made yet to incorporate a non-constant $\alpha_{n t h}$ in the analysis of the thermal contribution.

As an example I use the analysis of IC342 by Hummel and Gräve (1990). IC342 is the nearest, almost face-on spiral galaxy and also a relatively strong radio continuum source and therefore one of the best candidates to study. A disadvantage is its low galactic latitude which makes it hard to study for instance the $\mathrm{H} \alpha$ emission. For radii $<2 \mathrm{kpc}$ the emission is dominated by the central radio source. Most of the disk emission is well described by a plateau from $0 \rightarrow 5 \mathrm{kpc}$ and an exponential distribution beyond $\mathrm{r}=5 \mathrm{kpc}$. The scale lengths for the latter are 4.2 and $3.5 \mathrm{kpc}$ at 327 and $4750 \mathrm{MHz}$, respectively. After taking into account the relatively flat 
radio spectrum of the central radio source the spectral index between these two frequencies of the disk emission changes gradually from $\sim-0.65$ at $\mathrm{r}=0$ to $\sim-0.85$ at $\mathbf{r}=15 \mathrm{kpc}$. After correction for the thermal emission $\alpha_{n t h}$ is essentially constant with radius (of course partly a result of the assumption made to separate the thermal and non-thermal emission) and is $\sim-0.8$ and the $327 \mathrm{MHz}$ scale length can be considered as the scale length of the non-thermal emission $\left(\mathbf{R}_{n t h}\right)$.

Concerning the source distribution we have the two alternatives. Hummel and Gräve (1990) determined the radial distribution of the thermal emission. Beyond $r=5 \mathrm{kpc}$ its exponential scale length $\left(\mathrm{R}_{t h}\right)$ is $\sim 2.1 \mathrm{kpc}$. The radial distribution of the blue light has been measured by Ables (1971) and beyond 5kpc the exponential scale length $\left(\mathrm{R}_{\text {opt }}\right)$ is $\sim 3.4 \mathrm{kpc}$. This result $\mathrm{R}_{\text {nth }}>\mathrm{R}_{\text {opt }}>\mathrm{R}_{\text {th }}$ seems to be a more general result (Hummel, 1990).

In terms of propagation models it is not $R_{n t h}$ which is of importance but the scale lengths of the cosmic ray electrons and the magnetic field strength $\left(\mathrm{R}_{e}\right.$ and $\mathrm{R}_{B f}$, respectively). They are essentially not known, but assuming equipartition we obtain $\mathrm{R}_{e}=\left[\left(3-\alpha_{n t h}\right) / 2\right] \mathrm{R}_{n t h}$ and $\mathrm{R}_{B f}=\left(3-\alpha_{n t h}\right) \mathrm{R}_{n t h}$. This would make the cosmic ray scale length significantly larger than either $R_{t h}$ or $R_{\text {opt }}$, requiring for both source distribution hypothesis cosmic ray propagation in the radial direction. A typical radial propagation length in IC 342 would be $4 \rightarrow 5 \mathrm{kpc}$. An important constraint on the propagation and energy losses is the constancy or modest decrease of the spectral index with radius.

Apart from the uncertainties concerning the energy losses, source distribution, equipartition assumption there is the difficulty of the two-dimensionality of our observations. The radial distributions given in the literature (can) contain emission from both thin- and thick disk or halo components which can be discerned in at least some edge-on galaxies. The results on NGC891 and NGC4631 show that the radial extent of the thick disk or halo component is smaller than the radial extent of the thin disk component.

\section{RADIO HALOES}

The existence of haloes around spiral galaxies containing cosmic ray particles was first hinted at by cosmic ray physicists. Their evidence is based on measurements of the Be isotopes and the mean path length for cosmic rays of $\sim 5 \mathrm{~g} \mathrm{~cm}^{-2}$. A direct proof of the existence of a cosmic ray halo was obtained by Ekers and Sancisi (1977) when they detected a radio continuum halo around the edge-on spiral galaxy NGC4631 at a frequency of $610 \mathrm{MHz}$. A more detailed study of an edge-on galaxy was undertaken by Allen et al. (1978). They found two main components, a thin 
disk coinciding with the optically visible disk and a thick disk component (halo component) in NGC891.

\subsection{The Z-Distribution of the Radio Emission}

In Hummel (1990) I give a list of (well) studied edge-on spiral galaxies. This list can be extended by the results obtained from a recent survey of edge-on galaxies (Hummel et al. , 1990). For about 20 galaxies we have enough information to determine an half power width (FWHM of a gaussian distribution) of the radio distribution perpendicular to the plane (z-direction). A typical value for the FWHM, which is corrected for beam broadening and inclination effects, is $\sim 1 \mathrm{kpc}$ (distances consistent with $\mathrm{H}=100 \mathrm{~km} / \mathrm{sec} / \mathrm{Mpc}$ ). However, it appears that the FWHM is not (always) a good parametrization of the z-distribution. There is the complication of two (or more) distinct $z$-components but only very few galaxies have been observed in enough detail to try a decomposition. In addition, in the few cases for which observations with high enough resolution and sensitivity exist it turned out that the z-distributions are better described by an exponential than by a gaussian distribution.

The two cases that have been studied in most detail are NGC891 and NGC4631 (I assume distances to these galaxies of 7.2 and $7.5 \mathrm{Mpc}$, respectively). Allen and $\mathrm{Hu}$ (1985) noted that the z-distribution in NGC891 can be described by a gaussian thin disk and an exponential thick disk component. For the latter they found an exponential scale height $\left(\mathrm{Z}_{n t h}\right)$ of $0.9 \mathrm{kpc}$ at 1.4 and $4.9 \mathrm{GHz}$ out to their detection limit at $\sim 3 \mathrm{kpc}$. This is fully corroborated by recent and more sensitive measurements (see also Allen, this volume). Hummel et al. (1990) find a significant asymmetry in the sense that $Z_{n t h}=0.8$ and $1.0 \mathrm{kpc}$ on the $\mathrm{E}$ and $\mathrm{W}$ side, respectively. These scale heights are valid over a z-range of at least $4 \rightarrow 5$ scale heights. Similar results are obtained for NGC4631. Hummel and Dettmar (1990) find scale heights of 2.0 and $2.3 \mathrm{kpc}$ at $1.5 \mathrm{GHz}$ for the $\mathrm{N}$ and $\mathrm{S}$ side over a range of again $4 \rightarrow 5$ scale heights.

Apart from these large scale asymmetries NGC891 and NGC4631 show kpc scale structures in their haloes. The E side of NGC891 shows a clear peanut like morphology, and in the SW there is a prominent spur-like feature which extends to a z-height of $\sim 6.3 \mathrm{kpc}$. NGC4631 shows two main spurs, with lengths of $\sim 10 \mathrm{kpc}$, in the north.

\subsection{The Spectral Index Distributions}

Multi-frequency measurements with good enough resolution to determine the spectral index distribution ( $\alpha$-distribution) perpendicular to the plane of a galaxy have been made for NGC891 and NGC4631. The radio spectra of the total emission 


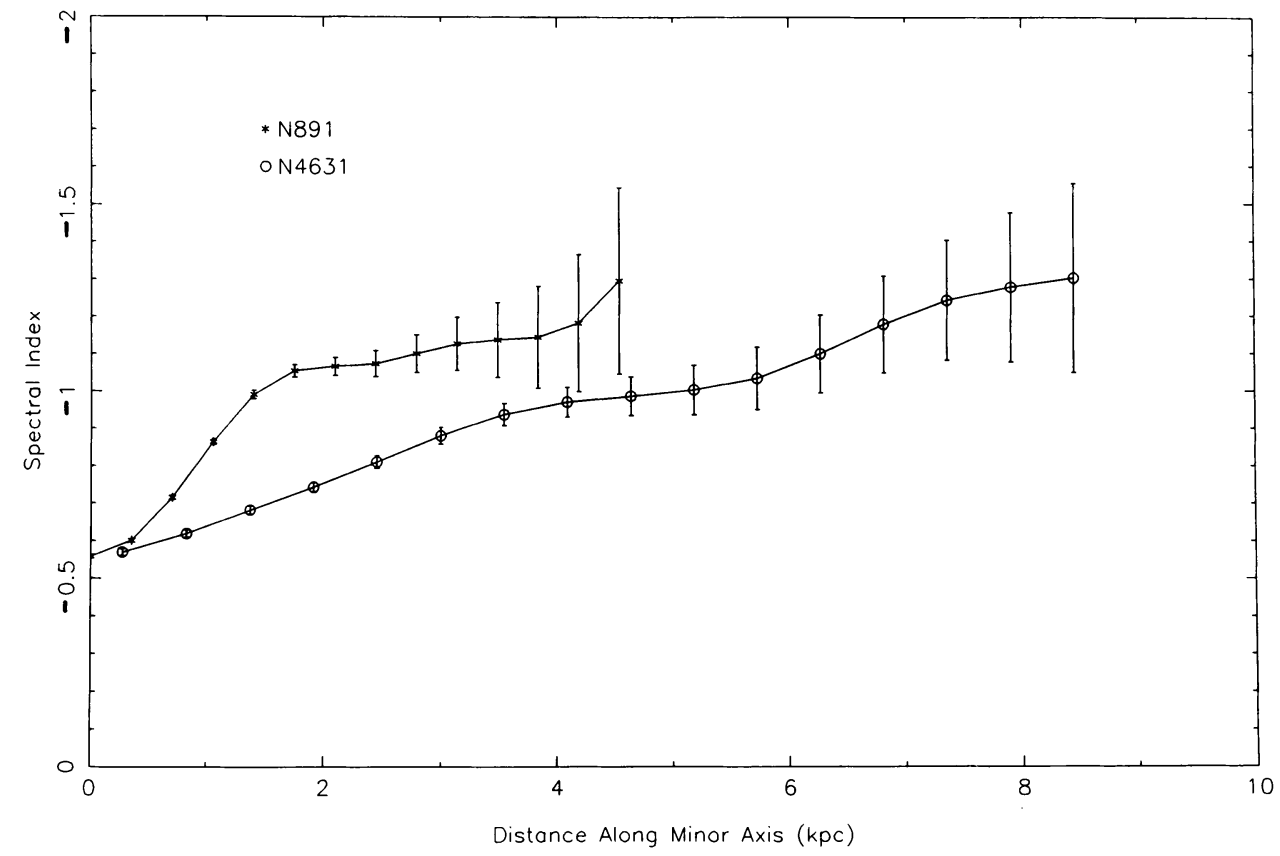

Figure 1: The spectral index distributions perpendicular to the optical major axis of NGC891 and NGC4631. They were determined from measurements at $610 \mathrm{MHz}$ and $1.5 \mathrm{GHz}$ with an angular resolution of $40^{\prime \prime}$.

from NGC891 and NGC4631 show a change in spectral index of $\geq 0.2$ and $\geq 0.3$, respectively. In both cases the "break" in the spectrum occurs at $\sim 0.8 \mathrm{GHz}$. Hence it appears that the relevant $\alpha$-distributions to construct are the ones using the "low" frequencies 327 and $610 \mathrm{MHz}$ and the "high" frequencies $1.5 \rightarrow 10.7 \mathrm{GHz}$. However, for various reasons I will use the measurements at $610 \mathrm{MHz}$ and $1.5 \mathrm{GHz}$ for the determination of the "high" frequency $\alpha$-distributions.

In Figure $1 \mathrm{I}$ show these "high" frequency $\alpha$-distributions for NGC891 and NGC4631. In case of NGC891 there is good agreement with the results given by Allen and $\mathrm{Hu}(1985)$, which were based on 1.4 and $5.0 \mathrm{GHz}$ measurements. The thin disk in NGC891 appears to have a $\alpha \sim-0.4$. The halo component has a rather constant $\alpha \sim-1.05$ from $\mathrm{z}=0$ to $3 \mathrm{kpc}$. Beyond that the spectrum steepens to $\alpha \sim-$ 1.3 at $\mathrm{z} \sim 4.5 \mathrm{kpc}$. The $\alpha$-distribution of NGC4631 does not show, like the brightness distributions, evidence for a major thin disk component. The spectrum gradually steepens from $\alpha \sim-0.55$ at $\mathrm{z}=0$ to $\alpha \sim-1.3$ at $\mathrm{z}=8.5 \mathrm{kpc}$.

The "low" frequency $\alpha$-distributions do not yet have such a good resolution as the "high" frequency distributions have and the work on these is still in progress. However, it is clear that the "high" frequency spectra in the planes of NGC891 and 
NGC4631 (i.e. on the major axis) are steeper by $\sim 0.25$ when compared to the "low" frequency spectra. This difference is also evident at larger z-distances, in particular on the W side of NGC891 and the N side of NGC4631. On these sides the "low" frequency spectral index is constant with $\mathrm{z}$ beyond $\mathrm{z}=3$ and $5.5 \mathrm{kpc}$ in NGC891 and NGC4631, respectively. On the E side of NGC891 and the S side of NGC4631 the "low" frequency spectrum steepens gradually from $\alpha \sim-0.4$ to $\alpha \sim-1.2$ at $\mathrm{z}=4$ and $9 \mathrm{kpc}$, respectively.

\subsection{The B-field in the Halo}

A very important parameter when dealing with cosmic ray propagation and which is accessable by radio continuum measurements is the magnetic field (B-field) strength and structure. This will be dealt with in more detail by Beck (this volume) but I cannot leave it unmentioned in the present context. Hummel et al. (1988) found a median B-field strength of $\sim 8 \mu \mathrm{G}$ in Sbc galaxies. After subtraction of the thin disk component, and assuming minimum energy conditions Hummel et al. (1990) find an average B-field strength in the haloes of NGC891 and NGC4631 of $\sim 8 \mu \mathrm{G}$ and $\sim 5 \mu \mathrm{G}$, respectively. Although there are ordered B-fields in these haloes the dominant field component is the random one.

The resulting half life times of the relativistic electrons radiating at $1.5 \mathrm{GHz}$ $(\mathrm{E} \sim 4 \mathrm{GeV})$ are then $\sim 410^{7}$ and $\sim 710^{7} \mathrm{yr}$, respectively, and assuming a diffusion coefficient $\mathrm{D}_{o}=10^{29} \mathrm{~cm}^{2} / \mathrm{sec}$ the typical diffusion length scales are $\sim 3.6$ and $\sim 4.8 \mathrm{kpc}$. However, because of the significant inverse Compton losses and the stronger B-field strengths in the planes of these galaxies these life times and diffusion length scales are upper limits. More realistic values, provided there is no reacceleration, are $\sim 3$ $10^{7} \mathrm{yr}$ and $\sim 3 \mathrm{kpc}$.

\section{THE CONSTRAINTS}

In this Section I give a brief summary of the more general results obtained from studies of the large-scale radio continuum structure of spiral galaxies and which are of importance for studies on the propagation of cosmic ray electrons. The results given here for IC342 and NGC891 may be considered as representative for spiral galaxies. However, it should be realized that the best studied galaxies are in general those with more than average radio emission. NGC4631, ironically, is most likely an exceptional case among the edge-on galaxies. The relevant findings are:

i) The large scale distribution of the non-thermal radio continuum emission is well described by exponential functions. In the radial direction this appears to be the case beyond a certain radius ( $\sim 5 \mathrm{kpc}$ in IC342). In that region $\mathrm{R}_{n t h}>\mathrm{R}_{\text {opt }}>\mathrm{R}_{t h}$. In 
the $\mathrm{z}$-direction there is evidence for at least two components. The thin disk component in NGC891 can be described by a gaussian or an exponential distribution. The thick disk component or halo is best described by an exponential distribution. For NGC891 and NGC4631 the $Z_{n t h}$ of the halo component is larger than $\mathrm{Z}_{\text {opt }}$. If the thin disk coincides with the star forming regions one can conclude that $Z_{n t h}>Z_{\text {opt }}>Z_{t h}$. How severe these constraints are on propagation models depends on the conversion from $\mathrm{R}_{n t h}$ and $\mathrm{Z}_{\text {nth }}$ to $\mathrm{R}_{e}$ and $\mathrm{Z}_{e}$ and on the source distribution.

ii) The radio spectra of NGC891 and NGC4631 clearly show a change of slope. The spectra become steeper with frequency and are consistent with a change in slope of $\Delta \alpha=0.5$ (i.e. $\Delta \gamma=1.0)$ with the break frequency near $0.8 \mathrm{GHz}(\mathrm{E} \sim 2 \mathrm{GeV})$. The low frequency measurements by Israel and Mahoney (1990) indicate that such a change of slope in the radio spectra could be rather common. The observed spectra do not support a confinement halo. A change in spectral index of $\Delta \alpha=0.5$ is expected for steady state systems from which the cosmic ray electrons can escape by diffusion or convection. Considering the half life time of the relativistic electrons of $\sim 310^{7} \mathrm{yr}$ the assumption of a steady state is valid for "normal" spiral galaxies. If the breaks in the radio spectra of NGC891 and NGC4631 result from convection by a galactic wind and adiabatic losses as envisaged by Lerche and Schlickeiser (1982) then the adiabatic cooling time is $\sim 410^{7} \mathrm{yr}$ and the $\mathrm{V}_{\infty}$ 's are $\sim 90$ and $\sim 150 \mathrm{~km} / \mathrm{sec}$, respectively.

iii) One of the questions to answer is whether the cosmic ray electrons propagate in a diffusive and/or convective mode. There are some indications that large scale convection is of importance in both radial and z-direction. In case of IC342 and NGC891 it is the constancy of $\alpha_{n t h}$ with radius and z-height which suggests that convection is needed while in NGC4631 it is the large z-extent itself. The arguments used involve time scales for the various energy loss processes and assumptions concerning the source distribution and the conversion from $\mathrm{R}_{n t h}$ and $\mathrm{Z}_{n t h}$ to $\mathrm{R}_{e}$ and $\mathrm{Z}_{e}$ and are therefore subject to large uncertainties.

In this respect note that with $\mathrm{D}=10^{29} \mathrm{~cm}^{2} / \mathrm{sec}$ and a life time for the cosmic ray electrons of $310^{7} \mathrm{yr}$ the diffusion length scale and the convection length scale are the same $(3.2 \mathrm{kpc})$, when the convection speed is $\sim 100 \mathrm{~km} / \mathrm{sec}$. This velocity is close to the Alfvén speed in a typical halo medium and the terminal velocity often associated with galactic winds. This means that on the basis of propagation lengths scales and time scales alone it is hard to rule out pure diffusion or pure convection propagation models.

In case of diffusion models with only synchrotron radiation losses the halo extent changes with frequency like $\nu^{-(1-\epsilon) / 4}$ where $\epsilon$ describes the energy dependence of the diffusion coefficient (e.g. Bulanov and Dogiel, 1974). For NGC891 and NGC4631 it is found that the halo extent changes like $\nu^{-0.3}$, resulting in $\epsilon \sim-0.2$. Cosmic 
ray studies indicate $\epsilon \sim 0.5$ (Ginzburg and Ptuskin, 1976), suggesting that diffusion models are not compatible with the halo extents as observed.

The presence of spur-like features in the haloes of NGC891 and NGC4631 and the peanut-like morphology of the E side of NGC891 can also be seen as manifestations of large scale convective motions of the cosmic ray electrons.

iv) A common results for NGC891 and NGC4631 is the difference of $\triangle \alpha \sim 0.25$ between the "low" and "high" frequency spectral indices in the planes of these galaxies. For NGC891 this difference is also present at larger z-heights (for NGC4631 this has not been determined yet). Also striking is the asymmetry in the "low" frequency $\alpha$-distributions. Both galaxies show a more or less constant $\alpha$ on the side which has the largest exponential scale height. On the other side the "low" frequency spectra steepen gradually.

The difference between the "low" and "high" frequency spectra at large z-heights in NGC891 strongly suggests that it is not caused by free-free absorption. Other ways to explain it are the importance of adiabatic losses and/or bremsstrahlung losses in the plane. Because of the low densities bremsstrahlung losses are of less importance at larger z-heights.

\section{CONCLUDING REMARKS}

From the radio continuum observations of edge-on spiral galaxies it is clear that cosmic ray electrons diffuse and convect away from their source distribution and that a typical propagation length is a few kpc. A large fraction of the cosmic ray electrons leave the source distribution, presumably associated with the thin star forming disk, perpendicular to the plane, hence forming a thick disk or halo component. This halo is not a confinement halo and the cosmic ray electrons can escape from the galaxy.

The results obtained for NGC891 and, in particular, NGC4631 on the spectra of the total radio emission, the "low" and "high" frequency spectra and the $\alpha$ distributions are consistent with galactic wind models in which adiabatic losses become important at $\nu<1 \mathrm{GHz}$ (Lerche and Schlickeiser, 1982; Hummel and Dettmar, 1990; Hummel et al. 1990). However, this is almost certainly not the only possible consistent model. Detailed modeling, including the various loss mechanisms and using the constraints given by the observations of the edge-on galaxies, is required. Observationally, high resolution, multi-frequency mapping of edge-on galaxies is important.

NGC4631 appears to show the strongest evidence for convection (possibly caused by a galactic wind) perpendicular to the plane. It is also an exceptional case concerning its radio continuum z-extent. Hummel and Dettmar (1990) suggest 
that this is due to the relatively high star formation rate in the plane of this galaxy and to the gravitational interaction NGC4631 is undergoing. Both circumstances would favour the onset of convection perpendicular to the plane of the galaxy.

An interesting relation that recently emerged in case of NGC891 is the one between the $\mathrm{H} \alpha$ emission and the radio continuum emission in the halo component. The $\mathrm{H} \alpha$ emission shows its largest z-extent in the northern half of NGC891 (Dettmar, 1990, also this volume). This might indicate that convection of cosmic ray electrons is already important at $\mathrm{z}$-distances of $\sim 2 \mathrm{kpc}$ and that it depends on the star formation activity in the disk.

\section{REFERENCES}

Allen, R.J., Baldwin, J.E., Sancisi, R.: 1978, Astron. Astrophys., 62, 397

Allen, R.J., Hu, F.X.: 1985, in New Aspects of Galaxy Photometry ed. J.L. Nieto, p293

Bicay, M.D., Helou, G., Condon, J.J.: 1989, Astrophys. J., 338, L53

Bulanov, S.V., Dogiel, V.A.: 1974, Astrophys. Space Sci., 29, 305

Dettmar, R.-J.: 1990, Astron. Astrophys., 232, L15

Ekers, R.D., Sancisi, R.: 1977, Astron. Astrophys., 54, 196

Ginzburg, V.I., Ptuskin, V.S.: 1976, Reviews of Modern Physics, 48, 161

Hummel, E.: 1990, in Windows on Galaxies, eds. G.Fabbiano, J.S. Gallagher and A.Renzini, Kluwer, Dordrecht, p141

Hummel, E., Dettmar, R.-J.: 1990, Astron. Astrophys., in press

Hummel, E., Gräve, R.: 1990, Astron. Astrophys., 228, 315

Hummel, E., van der Hulst, J.M.: 1986, Astron. Astrophys., 155, 151

Hummel, E., Davies, R.D., Wolstencroft, R.D., van der Hulst, J.M., Pedlar, A.: 1988, Astron. Astrophys., 199, 91

Hummel. E., Beck, R., Dettmar, R.-J.: 1990, Astron. Astrophys. Suppl. in press

Hummel, E., Dahlem, M., van der Hulst, J.M., Sukumar, S.: 1990, Astron. Astrophys., in press

Israel, F.P., Mahoney, M.J.: Astrophys. J., 352, 30

Klein, U.: 1990, in Windows on Galaxies, eds. G.Fabbiano, J.S. Gallagher and A.Renzini, Kluwer, Dordrecht, p157

Krause, M.: 1990, in Galactic and Intergalactic Magnetic Fields, eds. R.Beck, P.P.Kronberg and R.Wielebinski, Kluwer, Dordrecht, p187

van der Kruit, P.C.: 1977, Astron. Astrophys., 59, 359

Lerche, I., Schlickeiser, R.: 1982, Astron. Astrophys., 107, 148

Segalovitz, A.: 1977, Astron. Astrophys., 61, 59

Wunderlich, E.: 1989, $\mathrm{PhD}$ thesis University of Bonn 